\title{
La verve dans la mouvance rhétorique
}

Philippe Jousset

\section{(2) OpenEdition}

Journals

Édition électronique

URL : https://journals.openedition.org/recherchestravaux/705

DOI : 10.4000/recherchestravaux.705

ISSN : 1969-6434

\section{Éditeur}

UGA Éditions/Université Grenoble Alpes

\section{Édition imprimée}

Date de publication : 15 décembre 2014

Pagination : 15-27

ISBN : $978-2-84310-291-2$

ISSN : 0151-1874

\section{Référence électronique}

Philippe Jousset, "La verve dans la mouvance rhétorique », Recherches \& Travaux [En ligne], 85 | 2014, mis en ligne le 15 juin 2016, consulté le 29 octobre 2021. URL : http://journals.openedition.org/ recherchestravaux/705; DOI : https://doi.org/10.4000/recherchestravaux.705 
Philippe Jousset

Université Aix-Marseille

\section{La verve dans la mouvance rhétorique}

La verve fait partie de ces concepts qui n'en sont pas vraiment. Et qui, faute de définition sûre, sont d'un emploi largement intuitif. Chacun les entend à sa manière, les tire à soi et les fait servir à l'appui d'argumentations ad hoc. Reconnaissons d'ailleurs que c'est là le sort de la plupart des notions recyclées dans les sciences humaines après avoir longtemps été en circulation et s'être patinées dans l'usage courant. La multiplication des acceptions va en général de pair avec le flou de la notion, son ancienneté et la richesse de son spectre, et en résulte. Le mal n'est donc pas sans contrepartie, il n'est pas même certain que ce soit, sauf pour les techniciens de la chose intellectuelle qui veulent que chaque pièce soit exactement calibrée, un inconvénient. À raison même de son incertaine essence, la verve détient un potentiel métaphorique qui lui confère la faculté d'enjamber les domaines. Comme si le feuilleté du mot signalait celui-ci comme un "désignateur non rigide» (pour prendre le contre-pied de la définition de Kripke) de réalités indispensables à la civilisation des lettres.

Les préjugés suffisent pour la vie ordinaire, mais ils demandent le secours d'un plaidoyer dès qu'il s'agit de les confronter à d'autres préjugés pour prétendre l'emporter sur eux. Dans l'espoir de réduire malgré tout ces pétitions de principe, et tenter de mettre un peu d'objectivité dans cette question, nous passerons donc ces idées préconçues à l'épreuve d'un texte qui nous a spontanément paru appeler la qualification de verveux (puisque ce néologisme nous est permis par l'exemple de Sainte-Beuve qui en faisait l'application à Diderot). En procédant à de rapides vérifications in anima vili, nous trierons les motifs qui justifient qu'une simple impression de lecture, mais impérieuse, qui a cru trouver dans le mot de verve sa juste traduction, soit appuyée de raisons plus solides. Mais avant de chercher dans le texte en question un étayage à ce que nous paraît recouvrir le concept de verve, il est recommandé de recourir 
à quelques idées générales aussi consensuelles que possible, et l'intuition ne constituant pas un guide suffisant, c'est en resituant la notion dans une certaine constellation rhétorique qu'on pourra penser cerner les caractéristiques dont la présence nous paraît une condition sine que non pour s'autoriser à parler de verve et l'expliquer.

La première des caractéristiques les moins contestables qu'on puisse désigner comme inhérente à la définition de la verve paraît être l'abondance. Une abondance relative au genre pratiqué et aux possibilités de développement du motif. Si la copia dicendi à elle seule ne répond pas de la verve, si d'interminables énumérations ne suffisent pas à satisfaire à son cahier des charges ${ }^{\mathrm{I}}$, bref, si l'abondance ne se confond pas avec la verve, cette dernière n'existe pas sans copia ${ }^{2}$. Une verve maigre, une verve avare est une contradiction dans les termes. C'est probablement l'une des raisons de l'affinité de la notion, voire de sa rivalité, aux limites de la synonymie, avec le terme de veine. Le dictionnaire : «Inspiration vive et chaleureuse, imagination créatrice. Synon. veine», suivi de l'exemple : verve inépuisable (qui serait moins un pléonasme que l'indication d'une limite vers quoi la verve tend, à quoi elle montre une naturelle propension).

Probablement faut-il voir une conséquence de cette abondance dans le fait que la verve veut être répandue dans le texte. Elle ne se signale pas seulement par des traits isolés, en effet, tels que le mot d'esprit, la pointe..., tout ce qui relève de l'esprit ${ }^{3}$, mais suppose une forme de continuitét. Si on cherche à en parler en termes rhétoriques, on rapprochera cette exigence de ce que dit Cicéron de la cavillatio, cet enjouement ou badinage qu'il définit comme "verve répandue dans tout le corps du discours» et qu'il distingue de la dicacitas, ou causticité, "condensée en de brèves saillies`». Plus qu'une distinction,

I. Pensons au prologue du Tiers Livre par exemple, où la corne d'abondance métaphorise le texte, et à son allégorie imitative du «tonneau inexpuisible» qui a «source vive et vène perpétuelle».

2. On ajouterait dans le même champ sémantique les mots d'ubertas, varietas, divitiae, vis et facilitas, que nous retrouverons sous leur nom ou leur pseudonyme dans les développements qui suivent. L'antithèse de copia, techniquement, est l'inopia, ou sécheresse de style.

3. C'est pourquoi nous hésitons à utiliser le terme de Witz, associé dans la tradition romantique au Blitz et à l'hétérogène; or nous entendons ici l'esprit en tant que faculté, "conception", et non comme simple fabrique de concetti. Voir L'Absolu littéraire, présenté par P. Lacoue-Labarthe et J.-L. Nancy, Paris, Seuil, I978, p. 75.

4. L'expression «de verve» formerait une demi-exception. Surtout employée en peinture, elle suggère une exécution prompte, improvisée, et une inspiration qui trouve spontanément, en un premier jet, sa concrétisation. Cet "art de la notation cursive, de l'impression instantanée» à quoi fait allusion le "peindre de verve", permet de croquer le motif sur le vif, explique B. Vouilloux (L'Art des Goncourt. Une esthétique du style, Paris, L’Harmattan, 1997, p. 97-98).

5. Cicéron, De Oratore, 2, 218, cité par M. Blanco, Les Rhétoriques de la Pointe - Baltasar Graciàn et le conceptisme en Europe, Genève, Slatkine, I992, p. I63. 
c'est là une opposition en réalité, qui suscite des prises de position traversant la rhétorique tout entière et la partageant en deux grandes écoles, en conflit séculaire; on retrouverait par exemple, et au hasard, dans un registre grave, chez Marivaux, une opposition de même ordre (et une axiologie identique) entre le sublime de continuité d'un côté et les traits sublimes de l'autre ${ }^{6}$. La verve supposerait donc un certain tissu (cette «riche texture verbale» que les Latins nommaient contextus ${ }^{7}$ ) et pas seulement la présence de quelques bons mots et accidents particulièrement brillants, épars, dont le discours serait l'écrin ou le prétexte. Elle appelle une qualité d'homogénéité - un flux, du débit.

À défaut d'abondance, de continuité et d'une certaine homogénéité d'inspiration (pas obligatoirement ennemie de la variété, voire de la bigarrure, pourvu que les mêmes ingrédients soient continûment utilisés et pris dans un courant qui les porte et les unifie), on parlera plutôt de brio - qui figure parmi les synonymes de verve évidemment, mais s'en distinguerait justement par là. Le «morceau de bravoure» lui-même possède des dimensions a priori plus modestes, des limites plus facilement identifiables et, centré sur un seul motif, se trouve inséré dans un ensemble plus vaste. La verve est une prouesse qui se soutient et pas seulement une fusée?, une pyrotechnie nourrie et non l'explosion de quelques sonores pétards.

Pour cette même raison, la verve sera déficiente ou stérile lorsqu'elle se réduira à une déclinaison à base synonymique, chapelet de simples métaboles, expolitions ${ }^{\mathrm{ro}}$, périphrases, etc., soit : une façon de dire la même chose de plusieurs manières ${ }^{\text {II }}$. En avançant que la verve doit se soutenir, c'est ce que nous entendons : elle doit connaittre un renouvellement qui corresponde à un afflux de matière, une générosité d'inventio qui mette, par conséquent, en branle un art de la variation pas purement formel, lequel ne serait que l'application des deux procédés traditionnels d'expansion : "la dilatatio syntagmatique

6. Marivaux, «Sur la pensée sublime» [1719], Journaux et Euvres diverses, éd. F. Deloffre et M. Gilot, Paris, Garnier, 1969.

7. Cicéron, De partionibus oratoriae, 82. Voir T. Cave, Cornucopia. Figures de l'abondance au XVI siècle [1979], Paris, Macula, 1997, p. 29I.

8. Voir La virtuosité, $\mathrm{n}^{\circ} \mathrm{I} 28$ de Romantisme, 2005.

9. Au sens où R. de Gourmont parle des fusées de Voltaire.

Io. Métabole : «Succession de plusieurs termes sémantiquement proches ayant même nature et même fonction, et s'accompagnant parfois d'une gradation.» (P. Bacry, Les Figures de style, Paris, Belin, 1992). Expolition : «Figure macrostructurale d'amplification, qui consiste à développer, dans un discours, la même information sous plusieurs formes lexico-syntaxiques différentes. » (J. Mazaleyrat, G. Molinié, Vocabulaire de la stylistique, Paris, PUF, I989.)

II. Dans la rhétorique médiévale, la notion d'amplificatio était plus répandue que celle de copia. On peut imaginer que la désaffection pour l'amplificatio est à mettre en relation avec la montée de la notion de génie. 
- qui gonfle la syntaxe par la multiplication des composants - et l'amplificatio paradigmatique - portée, entre autres, par l'hyperbole ${ }^{\mathrm{r2}} »$. Une verve qui ne saurait que faire des gammes ressortirait à la rhétorique entendue en pire part, et manquerait à la fertilité, que suppose le concept, par exemple sous le nom d'ubertas qui, concurremment à la copia, est candidate à en subsumer l'ensemble des qualités. Cette notion d'ubertas, avant de désigner, chez Cicéron puis chez Quintilien, la richesse d'invention oratoire, et «l'abondance du style», se définit d'abord comme la fécondité du sol (chez le même Cicéron, par exemple, Tusculanes, I, II6).

L'idée de génie rôde dans ces parages. Loin d'être le produit d'une mimésis laborieuse, la verve manifeste une facilité par laquelle se reconnait sa naissance surnaturelle, s'alimentant directement aux sources vives de l'inspiration avec laquelle elle noue des liens privilégiés, ainsi qu'avec toutes les notions qui sont attirées dans l'orbite de ce mot mana. Nous songeons, pour parler latin - mais le français n'a pas jugé utile de lui trouver des équivalents - à l'afflatus, qui, là encore, est à la fois un élément naturel, souffle ou coup de vent ${ }^{13}$ puis, métaphoriquement, ce soudain afflux de l'impulsion créative, laquelle est communément attribuée à une influence divine. C'est souligner que la verve n'est pas un phénomène qui, du moins dans son principe, touche à la seule elocutio, à la seule surface du discours, à ses arrangements et procédés, mais relève aussi et d'abord d'une inventio, et puise son énergie ailleurs que dans le talent du rhéteur, du faiseur de phrases. Sans quoi sa richesse est purement de parure. Dans les termes du Dictionnaire de stylistique de Mazaleyrat et Molinié (où elle ne figure pas, tout comme elle est absente du Dictionnaire de poétique et de rhétorique de Morier ou de l'index de l'Histoire de la rhétorique dirigée par Marc Fumaroli), on parlerait de la verve comme d'une figure macrostructurale; elle "transcende techniques et matériaux spécifiques pour tendre vers un idéal d'énergie structurée, de discours en mouvement ${ }^{14}{ }^{14}$. Cette énergie est première, les mots n'en sont que la manifestation : la verve se reconnaît à celle-ci mais ne vit que de celle-là. Le lecteur remonte de l'une à l'autre : il sent l'énergie sous les mots. Et si elle fait volontiers appel à l'hypotypose, la verve ne donne pas seulement à voir : elle montre le muscle. Outre l'inventio, requise, et l'elocutio, à l'honneur, la verve met ainsi en branle un imaginaire de l'actio.

I2. Voir M. Levesque, "Une "syntaxe d'expressivité" ? Cyrano et la phrase ostentatoire», dans M. Levesque et O. Pédeflous (dir.), L'Emphase : copia ou brevitas? (XVI'-XVII siècles), Paris, Presses de l'université Paris-Sorbonne, 20Io, p. I45.

I3. C'est feng, le vent, qui traduit en chinois notre notion de style.

I4. T. Cave, Cornucopia, ouvr. cité, p. 33. 
C'est l'une des idées maitresses de l'ouvrage de Terence Cave, Cornucopia, que cette exigence pour la copia, divisée en copia rerum et copia verborum, que la seconde réponde de la première, que les res informent et gagent les verba. Sans quoi on a moins affaire à une véritable copia qu'à une simple vitiosa abundantia ou loquacitas $^{15}$. C'est ce qui explique que la verve soit un exercice à l'équilibre toujours menacé, comme nous aurons à l'approfondir un peu plus loin. Car on tombe aisément de verveux en verbeux. Chez Érasme particulièrement $^{16}$, on rencontre une conscience aiguë de cette nécessité d'une encaisse des verba, mais chez Plutarque déjà (qui lui consacre son traité De garruli$t_{t a t e^{17}}$ ) ou chez Pétrarque (De remediis), il est veillé à ce que la copia ne s'oublie pas et, ivre d'elle-même, ne sombre dans l'excès vers quoi son mouvement la porte si fatalement. L'inventio doit constamment régler l'elocutio pour ne pas tomber sous le coup d'une hubris, laquelle n'est pas blâmable seulement dans les mœurs, mais aussi dans l'éloquence, à l'image justement des mœurs dont doit faire preuve le vir bonus. L'importance accordée à ce qu'on appellerait aujourd'hui le Signifiant, et qui fait bien partie des propriétés essentielles de la verve, ne doit donc pas aller jusqu'à faire que les verba s'émancipent trop franchement des res, au point de les perdre de vue. Le discours tourne alors à vide, s'hystérise. Et l'étymologie qui rapporte la verve au latin verva, parole de Dieu, fût-elle en partie désacralisée sous son avatar nommé inspiration, rejoindrait alors le futile caprice, qui compte aussi parmi les sources étymologiques du mot ${ }^{18}$.

Il est une autre caractéristique à laquelle la définition de la verve échappe difficilement et qui découle en assez droite ligne de la personnalisation du discours (ou marquage) à quoi entraîne une prose qui se développe généreusement et se met en état de variation : elle signale la présence de son auteur. La verve ressortit à une mimesis qui ne peut faire oublier son mimeur. Alors qu'une prose idéalement neutre, ou simplement modeste, dénuée de propriété remarquable, une prose simplement suffisante, tend à faire oublier celui-ci. La verve n'est d'ailleurs pas spécifique au langage et à une manière d'écrire mais, ne se contentant pas d'enjamber les techniques (peinture, musique...), intéresse aussi la psychologie, pour indiquer un tempérament, fougueux,

15. Ibid., p. 34 .

16. On trouve quelques extraits de son De duplici copia verborum ac rerum commentarii duo (I5I2), traduits par J. Chomarat, dans ses Euvres choisies, Paris, Le Livre de Poche, I99I, et dans J. Chomarat, Grammaire et rhétorique chez Érasme, Paris, Les Belles Lettres, 198I.

I7. Garrulitas : caquet, bavardage, babil.

18. "Vx. Caprice, bizarrerie, fantaisie», selon Littré. Le TLF cite cette occurrence dans Maistre Pathelin où il semble que le mot ait à peu près le sens de délire (à rapprocher de la furor) : "Estes vous desvoyé? Recommancez vous votre verve?» 
enjoué, débordant d'énergie - ce qui la rend si incommode aux spécialistes qui se méfient des termes mal circonscrits, lesquels ne sont pourtant qu'un indice que la parole est prise avec nos représentations, avec l'imagination, etc. dans un tissu de relations multiples, non exclusivement livresques. De l'implication de l'auteur dans son texte à la mise en scène de soi, de celleci à l'histrionisme et au cabotinage (recourant à l'esthétique de la surprise, aux incongruités, ne reculant ni devant les coqs à l'âne ni devant les facéties - «Fantasyland, poil aux glandes»), il y a là aussi une pente que la verve, forcément tentée d'outrer le ton et d'accentuer l'aspect théâtral de sa diction, a tôt fait de dévaler.

On produirait pour illustrer cet aspect de la verve un tour comme celui-ci, qu'on lit aux premiers mots du texte que nous venons de citer :

Ça y est, c'est fait, j'en reviens, j’ai vu Eurodisneyland! Enfin vu. Entr'aperçu plutôt, ou ce petit jeu scénique énonciatif :

[...] et il vous faut du temps pour comprendre que ces noms [de lieux fleurant bon le terroir, sur les panneaux de signalisation] ne correspondent à rien. Enfin à rien. À des résidences, à des piazzas, à des parcs urbains, à des galeries marchandes avec vasques et lampadaires appropriés. C’est ce que je disais : à rien (p. I46).

Puisque nous avons commencé de citer ce texte que nous annoncions comme notre banc d'essai, avouons-le à présent qu'est esquissée la physionomie de la verve par combinaison des traits que nous avons reconnus (les principaux - abondance, continuité, densité, présence du narrateur - ne se retrouvent pas obligatoirement réunis mais la plupart doivent l'être pour que, à notre sens, on puisse parler de verve). Il s'agit du texte de Philippe Muray (I945-2006) intitulé «La colonie distractionnaire ${ }^{19}$ ».

Ce texte d'à peine une dizaine de pages évoque l'inauguration prochaine du parc de loisirs Euro Disney en banlieue parisienne. Romancier et chroniqueur, Muray a conduit, principalement sous forme de chroniques, une analyse argumentée et abondamment illustrée de la société contemporaine; il s'est rendu célèbre avant tout comme contempteur de l'Homo festivus, et il ne déroge pas à sa réputation avec ce texte dont la teneur polémique est patente.

Or, on concevra facilement que dans un genre offensif comme la satire, l'énergétique se donne plus volontiers carrière. La verve a une cible, et pas seulement un propos, une tête de turc parfois; l'opposition est son moteur, et

19. P. Muray, Désaccord parfait, Paris, Gallimard, coll. «Tel», 2000, p. I45-153. Le texte est daté de 1992. 
la détestation du monde-comme-il-va (à sa perte) est chez Muray un puissant mobile. À la fois le registre journalistique - d'un journalisme de haute volée et la nature satirique du texte favorisent sa qualité verveuse : parce qu'elle s'adresse à un public large et de par sa dimension pamphlétaire, ce réquisitoire feintement désinvolte s'apparente aux genres tribuniciens et en affecte la posture oratoire. C'est un élément supplémentaire, cohérent lui aussi avec ceux que nous avons rassemblés : la verve favorise ce qu'on appellerait les effets de diction, d'impromptu, un sentiment de direct (la "narration simultanée», les multiples retouches de l'auto-correction, etc.) qui a pour conséquence, en raccourcissant la distance de l'émetteur au lecteur, d'accroître la familiarité avec ce dernier. Rien d'étonnant, en effet, à ce que la verve soit fréquemment associée à une performance orale (on poserait que la verve est à l'écrit ce que la faconde est à l'oral ${ }^{20}$, l'oralité étant elle aussi un caractère dérivé de la mise au-devant de la scène de la première personne : Muray parle en son nom propre, et il est plus difficile d'imaginer une verve en troisième personne, au vrai, dès lors qu'elle suppose un fort investissement de celui qui s'énonce. L'auteur est compositeur et interprète, il joue sa propre partition. Et quand il confie celleci à un tiers, la marionnette dont il tire les fils parle un langage qui dissimule imparfaitement le manipulateur - songeons au Neveu de Rameau, l'un des meilleurs représentants fictionnels de la verve, qui aurait bien du mal à se faire passer pour autre chose que le double de Diderot lui-même.

L'inventivité, qualité inhérente à la verve, comme il a été établi, est sans doute la propriété la plus ostensible du texte de Muray et, la créativité venant à s'associer à l'abondance, elle se traduit le plus souvent par un exercice poussé de la variation : simple accumulation - dans cette évocation, par exemple, des bulldozers

poussant leurs tombereaux de cochonneries en couleurs, leurs dorures, leurs fanfreluches philanthropiques, leurs hôtels en glace à la framboise et leurs majorettes en chou à la crème bardées de gentillesse et de zoophilie.

Ou véritable figure argumentative : pour nous en tenir aux expressions par lesquelles l'auteur désigne sa cible, on relèverait ainsi les quelques équivalents suivants d'Eurodisneyland (rebaptisé depuis Disneyland Paris) :

la Ninive interactive, Euro-babylone (avec allusion historique, homéotéleute, composition lexicale),

20. T. Cave note que dans les écrits de J. Peletier du Mans (I5I7-I582), un théoricien important de ce qu'on n'appelait pas encore la poétique, la production de copia repose «sur ce qu'on peut imaginer comme une exacte superposition de l'oral et de l'écrit, comme un principe d'équivalence ou d'interchangeabilité» (Cornucopia, ouvr. cité, p. I80). 
la colonie distractionnaire du siècle en train de naître (périphrase contenant une paronomase in absentia $a^{21}$,

monstre (métonymie),

la Ville dont le prince est un con entouré de feux d'artifices (clin d'œil littéraire), jardin d'Éden, Meilleur des mondes, le Paradis (antiphrases par outrance, radicalisation, allusions)... On pourrait poursuivre, Muray faisant paraphrase de tout bois, soit : EuroDisneyland comme Royaume de la Souris sacrée, Cauchemar à thèmes, cheval de Troie de la version nursery du monde, étron, énorme bubon d'Eurolunapark, pays de la rigolade, monument élevé à notre effacement définitif, Eurosaloperie pur sucre et parpaing, le plus viral des divertissements américains, Pays des merveilles, grande mosquée de la Rigolade permanente, Eurocarnaval...

Muray récidive un peu plus loin lorsque le Château, pièce maîtresse du Parc d'attractions, sa cathédrale, vient à réaiguillonner son courroux (la verve, en effet, en concurrence avec les deux autres principales matrices, la redondance et la répétition, manifeste une structure fractale) : il vitupère ce Château,

Disneygraal de l'Idiotie au bois dormant! Leur Neuschwanstein en pistache! Tout stuc et bonbon! Poivrières, mâchicoulis, beffrois et merveilles! Oriflammes et fanfares! Ô tours divines! Étoile de mer! Chartres et Lourdes!

On voit que certaines dénominations (en compréhension ou en extension) relèvent du défoulement - lorsque la verve s'enivre d'elle-même pour ainsi dire, se prend à son propre jeu, varie pour varier (et alors, résonnez métaphores, jeux de rapprochement, synecdoques, amusettes (b-effrois)! que la Rhétorique générale du Groupe $\mathrm{Mu}$ trouverait certainement à ranger vaille que vaille dans une de ses quatre opérations logiques ${ }^{22}$ ); d'autres dénominations, toutefois, paraissent plus sérieuses et mieux justifiées en ce qu'elles sont étroitement reliées

2I. Nous avons étudié ce type de démarcation qui s’appuie sur une expression identifiable détournée pour produire une allusion dans «À propos des sur-énoncés» (colloque d'Arras, 20I3, à paraître). C'est ce que J.-C. Beacco désigne comme "la capacité à jouer sur la langue en s'en éloignant", qu'il donne pour une compétence d'écriture caractéristique de la verve (journalistique) à côté de la capacité à improviser, à «mobiliser du lexique très varié et peu usité» et à "créer des textes "chaleureux" " ("De la verve. À la recherche d'un idéal discursif ordinaire », Pratiques, $\mathrm{n}^{\circ}$ I39-I40, décembre 2008, p. I43). Le rifacimento est très prisé de Muray comme des pamphlétaires en général, exemple : «les Américains, eux, en vrais grands professionnels, vont nous apprendre de quel corso fleuri ils se chauffent». Le titre même de sa chronique est un bon exemple du procédé : il joue sur la ressemblance à la fois de pénitentiaireldistractionnaire et sur le mot colonie pour suggérer l'idée de «travaux forcés» du loisir, grégarisme, nouveau totalitarisme, etc. Ce genre d'allusion fait souvent appel à une culture, volontiers religieuse chez Muray, quand elle n'est pas littéraire qui, en même temps qu'elle constitue une forme de polyphonie réappropriée en nom propre, suppose connivence avec le lecteur, ainsi de : «Eurodisneyland morne plaine!», «La bataille de la Marne était perdue d'avance!», «En vérité, en vérité, je ne devrais pas plaisanter.»

22. Addition, suppression, fusion et déplacement sont les quatre "métaplasmes» exposés dans la Rhétorique générale du Groupe Mu (1970), Paris, Seuil, coll. «Points», 1992. 
au fond de l'argumentation et peuvent être considérées comme des éléments de définition de l'objet dont il est question ("auraient-ils jugé que nous étions plus mûrs, c'est-à-dire plus morts, que les Espagnols ?», ironise ainsi Muray lorsqu'il se demande pourquoi les Américains ont choisi la France; l'expression ici se révèle plus solide et plus grave, comme lorsque Muray écrit de la Souris sacrée : "son Royaume est de ce monde». Ou, politique : "Nos nouveaux maitres de droite comme de gauche, dans le fond de leur âme, s'appellent tous Mickey et Minnie.» On donnerait un autre échantillon de cet esprit qui ne cherche pas qu'à briller, emprunté à une chronique qui figure plus loin dans le livre, où est assumé le parti pris réactionnaire du projet :

Ainsi ai-je vite jugé qu'on ne pouvait plus écrire autrement que dans le sens contraire des aiguilles du monde (p. 342).

Ou encore, dans notre texte même, ce constat désabusé où le substantiel l'emporte également sur la gratuité :

Plus d'Histoire. Plus de temps. Plus que du passe-temps.

On retrouve ici les deux versants de la verve que nous évoquions supra: simple accumulation, lorsque les verba tendent à vivre leur propre destin en devenant insoucieux de leur rapport aux res - c'est alors sa dimension ludique, impulsive jusqu'au frivole, qui se montre, où la poésie, le lyrisme parfois, confinent à la parodie («Des bâtiments informes et mesquins se dressent sur l'horizon comme de vieilles molaires de béton essayant de mastiquer les nuages du ciel lourd.») Ou bien la multiplication des termes ainsi que la diversité des figures (jusqu’à l'adunaton ${ }^{23}$ ) répondent à la dynamique du propos, relancent le mouvement du texte, lui confèrent ses intensités, alimentent l'énergie polémique. Le langage figuré n'est plus alors un ornement plus ou moins superfétatoire, mais fait partie du «fond». Les exutoires, dans ce cas, représentent de nécessaires soupapes à l'excès. C'est ainsi que Muray allonge, dans une autre chronique, la liste des «emplois-jeunes» de Martine Aubry, "proposant des emplois destinés à l'améliorer vraiment, la vie des citoyens» :

Fracasseur de transistors, par exemple. Ou restaurateur du négatif. Dissuadeur de touristes dans les aéroports. Décourageur d'artistes contemporains. Entraveur de randonnées. Aggraveur de l'exception française. Décoordinateur polyvalent. (p. 334)

Dans notre texte, il fustige l'«Europe» coupable de normaliser les cigarettes Gitanes pour en faire

23. Adynaton : hyperbole impossible à force d'exagération. 
[...] ces cochonneries de cigarettes frauduleuses, culpabilisées, atrophiées, inhibées, neutralisées, domestiquées, anémiées, pusillanimes, vertueuses, bref, maastrichiennes, donc maastricheuses (p. 144),

où l'accumulation attendue se couronne par une ultime manipulation suffixale. Cette accumulation vaut pour un geste d'acharnement vengeur, de flagellation - il pleut des blâmes - et constitue donc un sens en acte dont une pragmatique des figures serait mieux à même de rendre compte qu'une classique rhétorique (on parle bien du fouet du style - et est-ce un hasard si les épithètes de Muray sont aussi nombreuses que les lanières du "chat à neuf queues»?). La gradation, qui forme une chaîne dont une extrémité est occupée par l'adjectif frauduleuses et l'autre par son antonyme vertueuses, dessine elle aussi un mouvement significatif où, au terme de l'énumération, le mot de vertueuses doit être entendu à contreemploi, ce qui exige un interprétant spécifique qui suppose une souscription du lecteur à l'axiologie sous-tendue par ce renversement des valeurs, imputable à «l'Europe» il va de soi, et présenté comme une conséquence des opérations précédentes : neutralisation, domestication, etc., dont elle est responsable. Là encore, on voit bien que la disposition du discours et sa tournure ont toute leur efficience, et peuvent être dits performatifs : ils valent comme imputation, procès dans l'épaisseur du discours. Le néologisme final n'est pas ornemental lui non plus et tient lieu de stigmatisation : la paronomase qui rapproche maastrichienne de maastricheuse entend identifier l'essence de Maastricht à l'escroquerie que la ville du traité incarne par antonomase (le résomptif qui introduit la conclusion confère in fine à l'énumération un statut de prémisse dans un syllogisme très personnel). Muray rend à Maastricht la monnaie de sa pièce en employant ses procédés : il truque, trafique, non pas en normalisant, lui, mais en se jouant de la norme, au contraire, pour réaffirmer son droit subjectif contre le Léviathan. La verve est ici protestation de l'individu singulier, attaché à ses cigarettes sans filtre, contre l'entropie bureaucratique, au moyen de la seule arme qui lui reste : l'usage singulier du langage, dans son excès revendiqué, dans son abus même, car si le paradoxe, dont l'amateur de verve est grand consommateur ${ }^{24}$, se montre insuffisant, la mauvaise foi est aussi permise, par l'abus même de l'adversaire, qui a commencé : elle y réplique.

Cette propension à exploiter la face sensible du langage, à jouer avec le Signifiant, quitte à se laisser entraîner de temps en temps à négliger le Signifié, n'est jamais totalement absente de la performance verveuse. Elle correspond à ce qu'on pourrait appeler une émulsion de la langue. On ne se

24. Exemple, où le paradoxe se résout en oxymore : "Tout doit être mis en ouvre pour que le monde soit au plus vite, et définitivement, visitable sans risques, nurserisé et perclus d'attractions.» (p. 3I) 
contente pas de recourir à celle-ci comme à un austère instrument intellectuel de désignation, mais elle se voit traitée en tant que matière signifiante en ellemême, qui participe du dictum et en est inséparable ${ }^{25}$. On vient de le voir, dans la charge par exemple, la véhémence n'est pas une surdétermination du propos mais une de ses dimensions. Dans les discours où la sécheresse n'est pas de mise ni la neutralité, où l'auteur "prend à cœur» l'opinion qu'il défend ou pourfend avec pugnacité celle qu'il attaque, prenant à partie un adversaire, la singularité débouche assez naturellement sur une floraison lexicale, quand ce n'est pas une débauche : les munitions sont prodiguées, le langage est à la fête, et la néologie s'y invite souvent, qui manifeste l'extraversion de la langue, son ouverture vers un dehors, un inouï auxquels la koinè ne suffit plus (il est question, par exemple, de gréviniser ce qui n'a pu être anéanti dans la capitale) ${ }^{26}$. On se trouve à l'étroit dans sa langue et, à force de la «solliciter», on l'excède. Si l'adversaire vient à manquer, il reste les moulins à vent pour penser mettre la langue en insurrection (à se demander s'ils ne font pas les meilleures cibles, en définitive). $\mathrm{Ou}$, si le bretteur est un peu comédien, le sens du parterre peut suffire, d'un public à étonner, à subjuguer, à éblouir, et qui applaudit in petto à la prouesse. Ou même, sans plus songer à la galerie, le rhéteur peut être porté à sacrifier au pur bonheur verbal sans finalité, et jouir simplement d'écrire en sur-régime ${ }^{27}$. Cela dé-foule (exalte l'individu).

En tant que l'étymologie fait tantôt remonter le mot à une forme dérivée de verba, séduisante dans sa simplicité même - la verve ainsi expliquée par son origine serait en quelque sorte le fonctionnement du discours à très haut rendement, la verve serait la parole par excellence ${ }^{28}$. D'abord lorsqu'elle rappelle sa dimension orale, la parole vive, la parole agissante, au mieux de sa forme, de ses formes : le terme de copia seul a longtemps été un synonyme d'éloquence. On l'a dit, la souplesse, la ductilité d'une telle notion sont celles

25. «Le pire qui puisse arriver à une langue, écrit M. de Gournay, c'est qu'elle manque d'étoffe», cité par I. Landy-Houillon, "Autour des synonymes : Copia et richesse de la langue française (XVI ${ }^{\mathrm{e}}-\mathrm{XVIII}{ }^{\mathrm{e}}$ siècle)», Entre philologie et linguistique, Paris, Classiques Garnier, 20I2, p. 30.

26. P. Muray est l'inventeur d'artistocrate (artiste qui prête serment d'allégeance aux politiciens et aux fonctionnaires), de rebellocrate (rebelle proche du pouvoir en réalité), mutin de Panurge, etc. Dans notre recueil, on relèverait : Khomeiny, «stéréotype du Mal absolu, diable sortant de sa boîte, imam d'Épinal» (p. I28).

27. T. Cave évoque "la dérive d'Agricola vers la jouissance d'écrire» (Cornucopia, ouvr. cité, p. I83).

28. Les dictionnaires attestent qu'on a pu anciennement user du mot - on cite un exemple pris chez Rutebeuf ou chez Guillaume de Lorris - comme d'un simple synonyme d'idée, chose à dire, discours. 
de tous les termes de culture qui, comme l'a expliqué Pierre Bourdieu, étant objectivement ambigus, surdéterminés ou indéterminés, doivent leur succès et leur survie précisément au fait qu'ils n'ont pas cessé d'être l'enjeu de luttes à propos de leur sens. Certaines conditions cependant doivent être remplies pour identifier la verve, dont nous avons tenté de réunir les moins discutables, en tentant de les resituer dans le temps long et la profondeur de champ de la rhétorique : l'abondance, qui peut être plus ou moins canalisée, plus ou moins exubérante; le déploiement figural, mais pris dans une continuité, avec les tours presque obligés qui caractérisent l'art oratoire (anaphore, accumulation, reformulation...), une forte présence de l'élocuteur ${ }^{29}$, et une virtuosité, qui donne souvent lieu à ce que les Latins, encore eux, nommaient du beau nom de festivitas, qui désigne l'enjouement, la langue en état de jubilation. C'est en ce point où la verve risque de tourner au pur exercice de style, le morceau de bravoure étant le spécimen le plus connu et le plus achevé de ce péril.

Le jugement de valeur intervient ici qui départage depuis l'aube de la rhétorique, pour le dire vite, l'asianisme et l'atticisme, la sophistique et l'idéalisme, la mythologie de l'Orient opulent et fleuri et de l'Occident plus mesuré et maître de soi... La verve, lorsqu'elle n'est pas flétrie comme un défaut, une dépravation, réalise cet équilibre dont nous avons parlé entre les verba et les res, lequel menace incessamment de se rompre :

Si à chaque instant l'on n'y prend garde, prévenait Sainte-Beuve, il y a là, quelles que soient les belles choses qu'on dit, et même plus on dit de belles choses, une déviation morale très-prochaine, une tentation qui fait aisément qu'on s'occupe bien moins de les penser et de les pratiquer que de les dire, que d'y inscrire et d'y enchâsser éternellement son nom comme Phidias dans le bouclier de sa Minerve ${ }^{30}$.

Ubi uber, ibi tuber dit un adage latin : où il y a abondance, l'enflure menace ${ }^{31}$, qui trouve également une application à la verve, indulgente aux excès. Elle est alors cette tare qui vient s'ajouter inopportunément, voire indécemment, au fond des sujets graves, genre avec lequel elle s'accommode difficilement, les corrompant $^{32}$. Pour tous ceux qui se font de la littérature une idée sérieuse, la

29. Cette présence met la notion de verve au contact de celle, fondamentale pour la rhétorique, d'evidentia ou enargeia, laquelle touche à la vaste question des moyens que le discours met en œuvre pour atteindre son lecteur, faire effet sur lui, l'impressionner.

30. Sainte-Beuve, Port-Royal, Paris, Gallimard, coll. «Bibliothèque de la Pléiade», t. II, I954, p. $572-573$.

31. Apulée, Florida, i8.

32. Sur un sujet sérieux entre tous, on mentionnerait ainsi, dans la liste que N. Cru dresse des «principaux artifices» auxquels ont recours les écrivains pour transmettre l'expérience de la guerre et dont il convient, éthiquement, de se défier : les «morceaux brillants qui manifestent la verve, l'humour, les traits d'esprit, la virtuosité verbale de l'auteur» (F. Rousseau, "Comment 
verve reste une tentation qui ne sera tolérée, et dans certaines étroites limites, que si elle est justifiée par la richesse des res qu'elle met en ouvre. Pour ceux-là l'émulation figurale des res par les verba doit garder la richesse du développement dans l'orbite de l'argument et ne pas tourner au profit des secondes, laisser la plume prendre son indépendance, "lâcher bride à l'écriture ». La jouissance pour elle-même est traditionnellement perçue comme diabolique.

Mais en deçà d'une morale de l'écriture ou d'une hygiène des lettres, et audelà d'une très ancienne opposition - Isocrate contre Démosthène : le premier qui ne pense qu'à «l'arrangement des mots», compose "des discours fleuris et efféminés, des périodes faites avec un travail infini pour amuser l'oreille", pendant que Démosthène, trop vivement touché des intérêts de sa patrie, n’a garde de s'amuser à tous ces jeux d'esprit ${ }^{33}$ - c'est d'un véritable partage anthropologique que témoignent les évaluations dont la verve fait l'objet : ce n'est pas seulement un goût dont disputent les partisans d'une expression filiforme, les «jansénistes» de la prose, les cérébraux, les concentrés, etc., en butte aux prodigues, sanguins, aux adeptes des ressources plantureuses, "florides", etc. Les uns, confiants dans la raison et la rigueur, défendent dans l'ordre du discours une économie de pénurie, ou d'autarcie, les autres, plus adonnés au chant, pratiquent une économie de type somptuaire - l'inusable fable des fourmis et des cigales, en somme.

écrire la guerre? L'affaire Norton Cru", dans C. Dornier et R. Dulong [dir.], Esthétique du témoignage, Paris, Éditions de la Maison des Sciences de l'Homme, 2005, p. 8-9).

33. Voir Fénelon, Dialogue sur l'éloquence en général et sur celle de la chaire en particulier (I7I8), dans Euvres, Paris, Gallimard, coll. «Bibliothèque de la Pléiade», I983, p. 8-9. Ce texte présente l'intérêt de mettre une fois de plus en présence et de dialectiser, dans un dialogue à trois, différentes positions à l'égard de l'éloquence. 